\title{
Investigation of a Random-Fractal Antenna Based on a Natural Tree-Leaf Geometry
}

\author{
Hatem Rmili, ${ }^{1}$ Donia Oueslati, ${ }^{2}$ Imen Ben Trad, ${ }^{3}$ Jean Marie Floch, ${ }^{3}$ Abdullah Dobaie, ${ }^{1}$ \\ and Raj Mittra ${ }^{1,4}$ \\ ${ }^{1}$ Electrical and Computer Engineering Department, Faculty of Engineering, King Abdulaziz University, P.O. Box 80204, \\ Jeddah 21589, Saudi Arabia \\ ${ }^{2}$ ICTEAM Institute, Université Catholique de Louvain, Louvain-la-Neuve, Belgium \\ ${ }^{3}$ IETR, INSA, 20 Avenue Buttes des Coësmes, 35043 Rennes, France \\ ${ }^{4}$ EMC Lab, Electrical and Computer Engineering Department, University of Central Florida, Orlando, FL 32816, USA
}

Correspondence should be addressed to Hatem Rmili; hatem.rmili@yahoo.fr

Received 12 June 2017; Revised 14 September 2017; Accepted 11 October 2017; Published 19 November 2017

Academic Editor: N. Nasimuddin

Copyright (c) 2017 Hatem Rmili et al. This is an open access article distributed under the Creative Commons Attribution License, which permits unrestricted use, distribution, and reproduction in any medium, provided the original work is properly cited.

In this paper, we investigate a new printed antenna based on the 2D image of a fractal tree-leaf geometry by studying the effect of the irregular boundary of the proposed antenna on its radiation characteristics. Both the impedance matching properties and the radiation patterns of the antenna are studied over the frequency band 1-6 GHz. Four configurations are designed by increasing the complexity of the structure, which ranges from iteration 0 to iteration 3 . The fractal properties of the proposed tree-leaf antenna are then compared to those of a conventional fractal antenna with smooth edges. Following this, the proposed antennas are fabricated and characterized experimentally. Finally, results are analyzed and discussed, and a practical application for this new type of antennas is proposed.

\section{Introduction}

The fractal concept can be applied to model natural objects characterized by geometrical properties such as self-similarity and scale invariance [1]. In the antenna field, the fractal concept has been used to produce innovative antennas with miniature shapes and multiband and/or wideband behaviors [2-5]. Fractal antenna geometries can be classified into two categories: deterministic and random. Numerous studies of deterministic antennas for which the self-similarity property can be described by a scaling power law have been carried out in the literature $[2,3]$. However, the same cannot be said about random type of fractal geometries [6], which do not lend themselves to a convenient mathematical description. A few studies [6-9] have recently been devoted to nature-inspired fractal antennas, whose properties have been compared to those of the conventional type of fractal antennas. In [9], a fluid-jet photo was used to design an irregular fractal-type of the printed antenna, which was shown to have a multiband behavior with radiation properties that are highly influenced by the irregular nature of the patch contour. In fact, the measured radiation patterns were found to exhibit some suck-outs (dips) and irregularities, which may be attributed to the excitation of high-order modes. In [6-8], fractal-metallic deposits obtained by electrochemical deposition were also used to investigate $2 \mathrm{D}$ and $3 \mathrm{D}$ random-fractal antennas. Again, it was found that the fractal geometry has a high impact on the antenna performance.

In this study, we have used the geometry of the Populus tremuloides leaf, which is a common North American species (Colorado Rocky Mountains, USA) [10] to design a new antenna and investigate the effect of the leaf contour and nervure network on the performances of the structure. First, we have numerically studied the $2 \mathrm{D}$ leaf geometry using MATLAB in order to detect its contour and nervure network. Following this, we have exported the processed image to the electromagnetic software Ansys-HFSS to simulate the 
radiating elements of the patch antenna. We have considered a total of four configurations with different levels of complexity of the nervure network. Finally, we have simulated the designed structures and have also characterized them experimentally. The bioinspired designed antenna which is characterized by complex fractal shape and unique electromagnetic signature can be used to secure chipless RFID systems [11]. In fact, RFID tags based on irregular fractal shapes inspired from nature are difficult to replicate by an unauthorized reader, especially when they are printed with invisible conductors which is important for authentication, anticounterfeiting, and security applications.

\section{Design and Simulation Procedures}

2.1. Image Acquisition and Processing. The leaf was soaked in a $5 \%(w / v)$ sodium hydroxide water solution for several days before being rinsed with water and bleached in 5\% $(w / v)$ sodium hypochlorite water solution for 5 minutes. After that, the leaf was transferred via an ethanol dehydration series $(50 \%, 100 \%)$ to a $0.1 \%(w / v)$ safranin ethanol staining solution for 1 hour before being cleaned using ethanol for 2 hours. The leaf was then transferred via series (50\% toluene, $50 \%$ ethanol) to $100 \%$ toluene and permanently mounted on glass slides using Fisher Permount medium.

Veins network of the young quaking aspen leaf (Populus tremuloides) was magnified four times; in fact, the image of the leaf shown in Figure 1 was obtained by stitching multiple frames from a $4 \mathrm{x}$ deconvolution microscope with transillumination and coupled to a $1024 \times 768 \mathrm{px}^{2}$ CCD camera. The image contrast was enhanced by keeping only the green channel of each image and then performing a contrast limited adaptive histogram equalization. The final image scale was 430 pixels per millimeter. We hand-traced all veins in each usable image using GIMP software.

2.2. Antenna Design. The contour of a radiating patch is an important parameter which affects the radiation properties of the antenna. Since the radiating patch is obtained from a 2D complex fractal image, MATLAB was used for processing the image as well as for reproducing the fractal patch, which was simulated by using the software Ansoft-HFSS (version 2015). We have used MATLAB to detect automatically the complex contour of the leaf image, then by using an appropriate code we have determined the coordinates of all points defining the boundaries of the image. Finally, a visual basic script (vbs) was developed to export final data into HFSS and reproduce the fractal image [12].

We have investigated the structure numerically by determining its multiple resonant frequencies, surface currents, and the radiation properties such as radiation patterns and gain values. Owing to the high complex nature of the contour (Figures 2(c) and 2(d)), only iteration 0 (Figure 2(a)) and iteration 1 (Figure 2(b)) of the structure were simulated numerically to determine all of their resonant frequencies and to derive the performance characteristics of the antenna at these frequencies.

We have adopted the strategy of progressively increasing the complexity of the design when analyzing the antenna

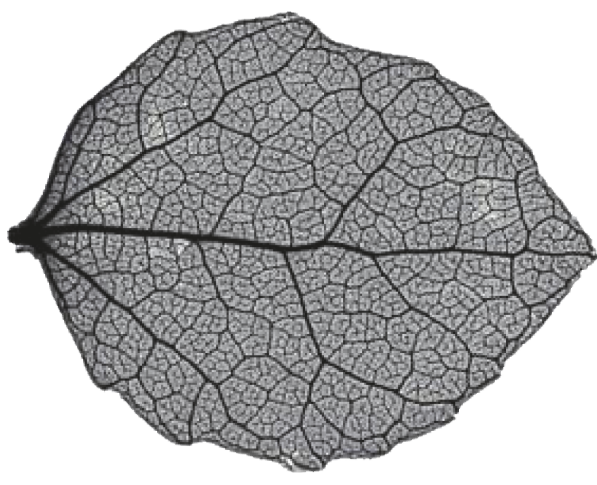

FIgURE 1: Image of the considered fractal tree-leaf.

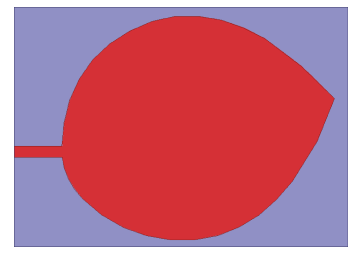

(a)

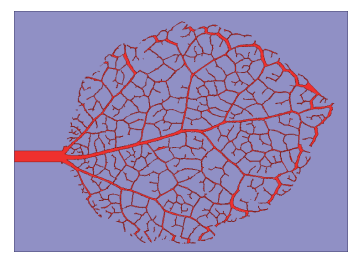

(c)

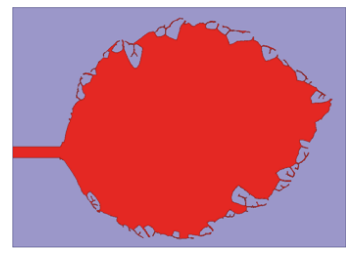

(b)

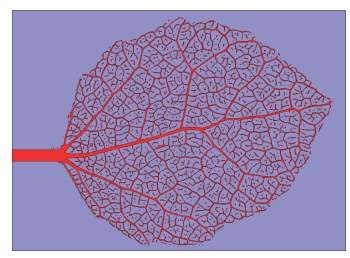

(d)
FIGURE 2: Schemas of the designed antennas: (a) iteration 0 (IT0), (b) iteration 1 (IT1), (c) iteration 2 (IT2), and (d) iteration 3 (IT3).

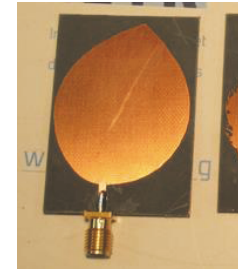

(a)

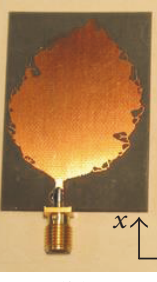

(b)

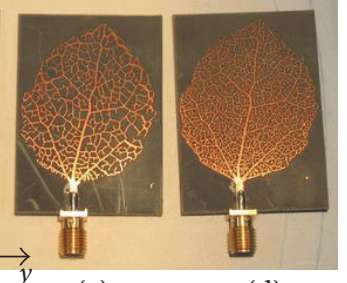

(c) (d)
FIgURE 3: Antenna prototypes: (a) iteration IT0 (A0), (b) iteration IT1 (A1), (c) iteration IT2 (A2), and (d) iteration IT3 (A3).

behavior. Four antennas denoted by A0, A1, A2, and A3 have been designed based on the fractal leaf image as depicted in Figure 3. First, we have designed the basic structure A0 by considering a smooth contour of the image, which closely follows the outer shape of the leaf. Then, we have taken into account the contour details and irregularities associated with the antenna A1. Next, we have added the primary nervure network of the leaf to design the antenna A2, retaining only nervures whose thickness exceed $0.5 \mathrm{~mm}$. Finally, for the antenna A3, we 


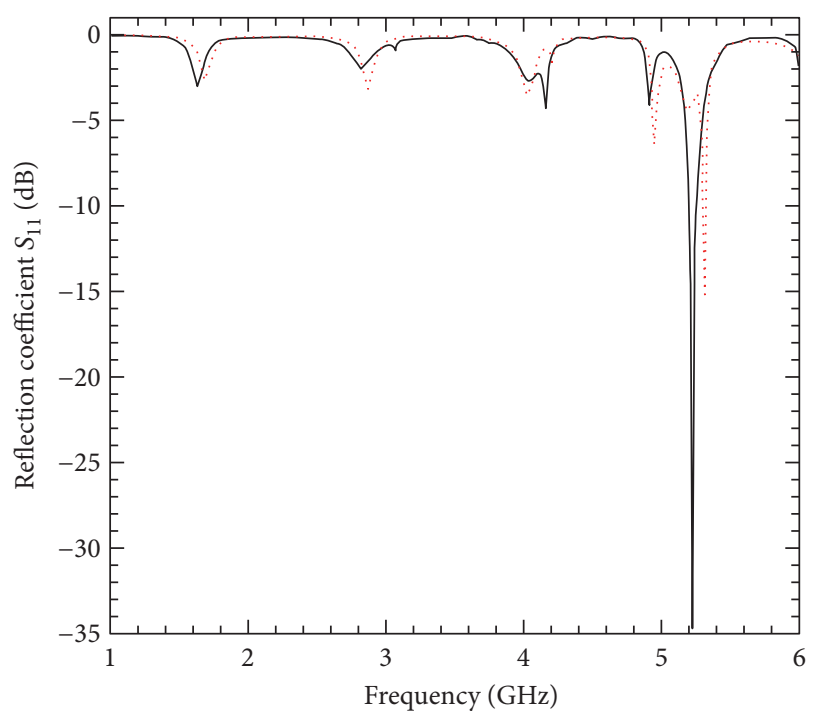

— Measurement Simulation

(a)

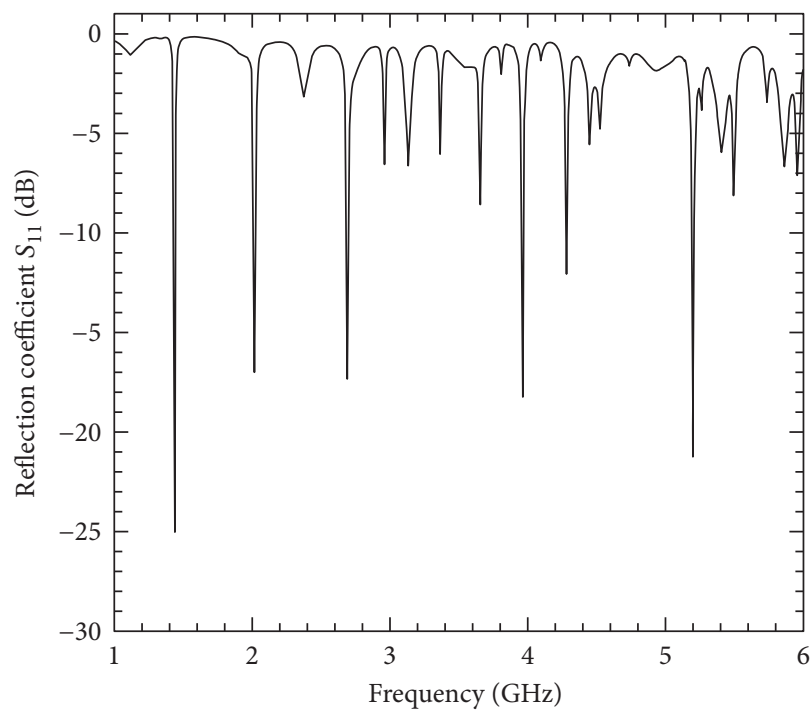

(c)

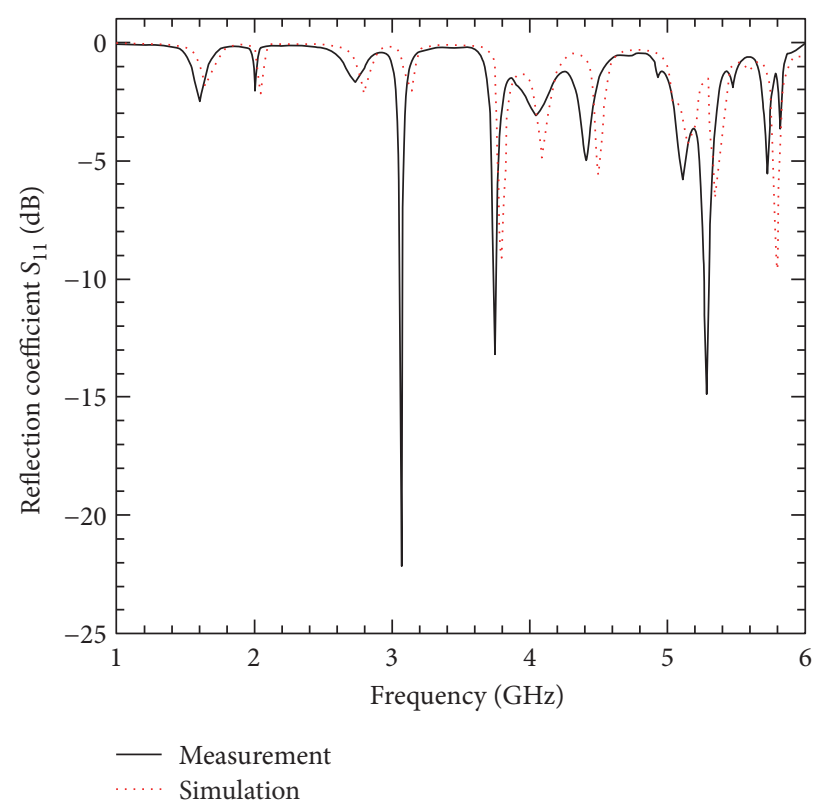

(b)

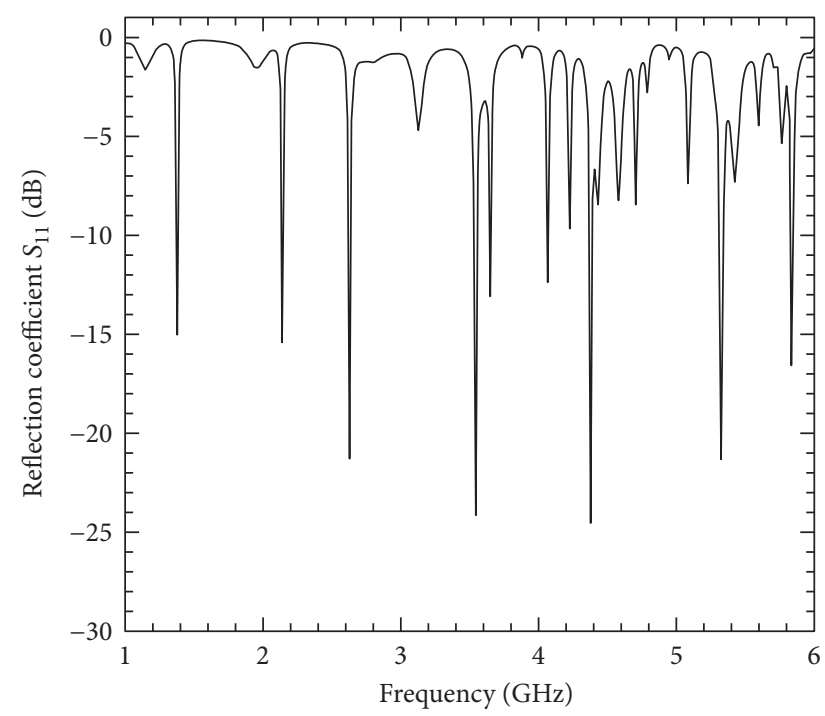

(d)

FIGURE 4: Simulated (red) and measured (black) reflection coefficient $S_{11}$ : (a) IT0, (b) IT1, (c) IT2, and (d) IT0.

have considered small nervures with a thickness less than $0.5 \mathrm{~mm}$, so that resulting geometry is quite close to that of the original leaf which we are approximating.

Next, we have excited the four antennas by using a $50 \Omega$ microstrip line, which is $13 \mathrm{~mm}$ in length and whose width is $3 \mathrm{~mm}$. Both the leaf-shaped patch and the microstrip line are printed on a duroid substrate with dimensions $60 \times 90 \mathrm{~mm}^{2}$, thickness $0.8 \mathrm{~mm}$, and relative permittivity of 2.17. The bottom face of the substrate is metalized to serve as a ground plane.

We have simulated only antennas related to iterations IT0 and IT1 because for the other iterations IT2 and IT3, the number of image detail is very high which increases significantly the number of finite elements (tetrahedrons) where Maxwell equations should be solved and exceeds the capacities of all our available numerical facilities.

\section{Results and Discussions}

We have investigated, both numerically and experimentally, the antennas shown in Figure 3, and the results are presented below.

3.1. Reflection Coefficient $S_{11}$. The return loss evolution versus frequency of the four proposed antennas over the band $1-6 \mathrm{GHz}$ is shown in Figure 4. We have analyzed the presented results and have found that they have a multifrequency behavior: 4 resonance frequencies for the antenna 
TABLE 1: Main measured resonance frequencies.

(a)

\begin{tabular}{lcccc}
\hline & \multicolumn{5}{c}{ A0 } & & \\
\hline$F(\mathrm{GHz})$ & 1.64 & 2.8 & 4.05 & 5.25 \\
$S_{11}(\mathrm{~dB})$ & -2.8 & -2.1 & -3.7 & -34.5 \\
\hline
\end{tabular}

(b)

\begin{tabular}{lccccccc}
\hline \multicolumn{7}{c}{ A1 } \\
\hline$F(\mathrm{GHz})$ & 1.6 & 2.76 & 3.07 & 3.73 & 4.41 & 5.28 & 5.72 \\
$S_{11}(\mathrm{~dB})$ & -2.23 & -2 & -22 & -13.04 & -5 & -14.66 & -5.5 \\
\hline
\end{tabular}

(c)

\begin{tabular}{lccccccc}
\hline & \multicolumn{5}{c}{ A2 } \\
\hline$F(\mathrm{GHz})$ & 1.45 & 2.03 & 2.38 & 2.7 & 3.97 & 4.29 & 5.20 \\
$S_{11}(\mathrm{~dB})$ & -25.0 & -16.9 & -3.14 & -17.3 & -18.3 & -12.0 & -21.2 \\
\hline
\end{tabular}

(d)

\begin{tabular}{lccccccccc}
\hline \multicolumn{10}{c}{ A3 } \\
\hline$F(\mathrm{GHz})$ & 1.38 & 2.15 & 2.62 & 3.13 & 3.55 & 4.06 & 4.37 & 5.32 & 5.85 \\
$S_{11}(\mathrm{~dB})$ & -15.0 & -15.4 & -21.2 & -4.7 & -24.1 & -12.3 & -24.5 & -21.3 & -16.5 \\
\hline
\end{tabular}

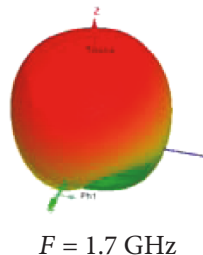

$F=1.7 \mathrm{GHz}$

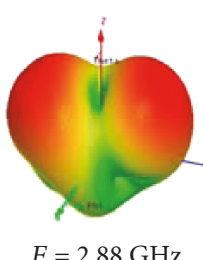

$F=2.88 \mathrm{GHz}$

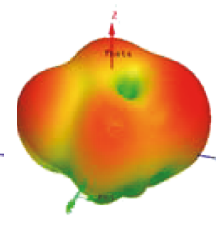

$F=4.03 \mathrm{GHz}$

(a)

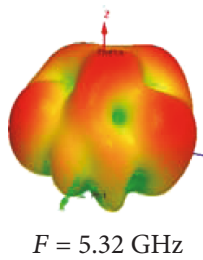

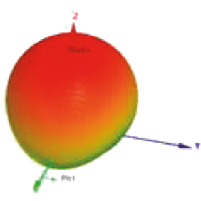

$F=1.66 \mathrm{GHz}$

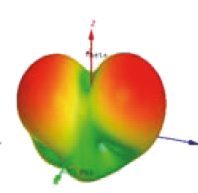

$F=2.80 \mathrm{GHz}$

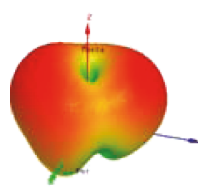

$F=3.13 \mathrm{GHz}$

(b)

FIGURE 5: Simulated 3D-radiation patterns at resonant frequencies for antennas: (a) A0 and (b) A1.

A0 (for IT0), 7 for A1 (for IT1), 10 for A2 (for IT2) with better input impedance matching, and 13 for A3 (for IT3). The distribution of resonance frequencies depends on the distribution of lengths over the fractal tree-leaf antenna. In fact, the more we increase the complexity of the contour and of the nervures network, the more resonance frequencies we obtain. As a consequence, we obtain a rich spectral structure with many narrow frequency bands and good input impedance matching within the measured frequency range 1-6 GHz. Furthermore, we can notice a good agreement between measurement and simulated results as illustrated in Figures 4(a) and 4(b). Table 1 summarizes the main results we have obtained for the measured resonance frequencies. The high complexity of the fractal patch antenna and its multi- and narrowband behaviors offers it a unique electromagnetic signature, permitting its use to secure chipless RFID systems [11].
3.2. Radiation Patterns and Gain. The simulated radiation patterns for antennas $\mathrm{A} 0$ and $\mathrm{A} 1$ at resonant frequencies within the range $800 \mathrm{kHz}-6 \mathrm{GHz}$ are given in Figure 5 .

We have used the anechoic room Stargate 32 designed by the society SATIMO operating within the range $800 \mathrm{kHz}-$ $6 \mathrm{GHz}$ to measure the radiation patterns. The antenna under test is placed in the center of the chamber. The experimental setup consists of 32 bipolarized probes placed on an arch situated above the antenna, then by simple rotation of the antenna under test in the azimuth-plane $(0<\phi<2 \pi)$, we get rapidly the radiation patterns at all the frequencies from the range $800 \mathrm{kHz}-6 \mathrm{GHz}$. Measurements are realized first in the near-field region, then by using an adequate software developed by SATIMO, we can determine the radiated farfields and then the far-field radiations patterns.

The measured $3 \mathrm{D} E_{\text {total }}$ radiation patterns at the resonance frequencies of the four antennas A0, A1, A2, and A3 


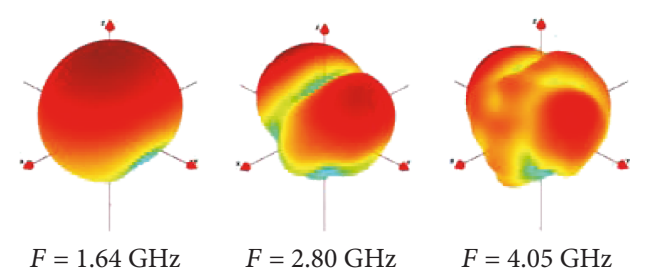

(a)

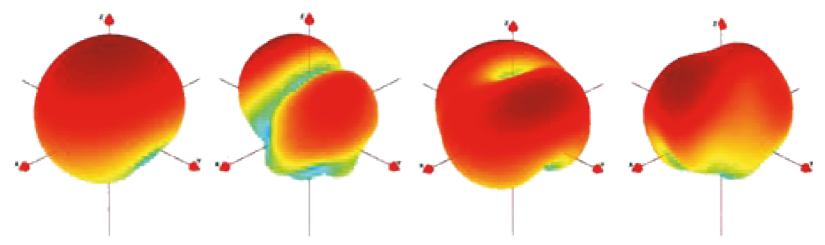

$F=1.60 \mathrm{GHz} \quad F=2.76 \mathrm{GHz} \quad F=3.07 \mathrm{GHz}$

$F=3.73 \mathrm{GHz}$

(b)
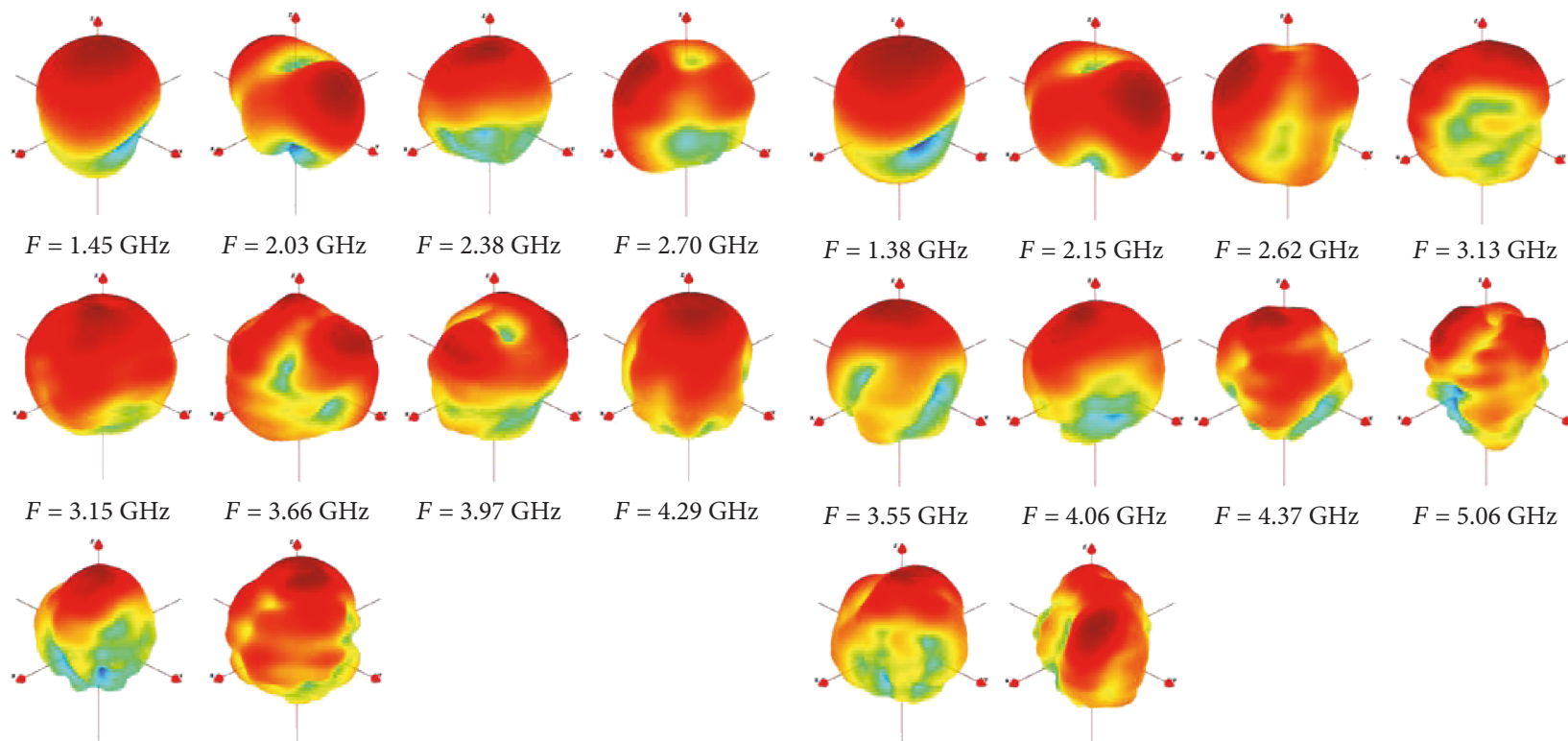

$F=3.97 \mathrm{GHz}$

$F=4.29 \mathrm{GHz}$

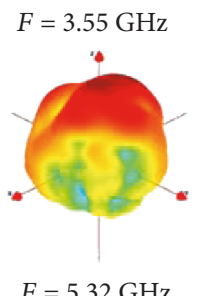

$F=4.06 \mathrm{GHz}$

$F=4.37 \mathrm{GHz}$

$F=3.13 \mathrm{GHz}$

$F=5.20 \mathrm{GHz} \quad F=5.49 \mathrm{GHz}$

$F=5.32 \mathrm{GHz}$

$F=5.85 \mathrm{GHz}$

(c)

(d)

FIgURE 6: Measured 3D-radiation patterns at resonant frequencies for antennas: (a) A0, (b) A1, (c) A2, and (d) A3.

are depicted in Figure 6. Comparison with simulated patterns for antennas $\mathrm{A} 0$ and $\mathrm{A} 1$ is satisfying. For the iterations IT0 and IT1, the antenna A1 differs from the antenna A0 only by the irregularity of its contour, which may explain why their radiation patterns, shown in Figures 6(a) and 6(b), exhibit a great deal of similarity at their corresponding resonance frequencies, namely, $F=1.64 \mathrm{GHz}$ (IT0) and $F=1.6$ $\mathrm{GHz}$ (IT1) and $F=2.8 \mathrm{GHz}$ (IT0) and $F=2.76 \mathrm{GHz}$ (IT1).

Also, a similar phenomenon is observed for iterations IT2 and IT3. Because of the similarities of the structure shapes (with higher complexities of the nervures for A3), the antennas A2 and A3 behave in the same way at lower frequencies, as may be seen from Figures 6(c) and 6(d). These antennas have random behaviors, which may be attributed to the randomness of the fractal structure itself and the asymmetry of the fractal shape. Good realized gain is achieved for antennas A0 and A1.

In Figure 7, we present both simulated and measured gain evolution over the frequency band $1-6 \mathrm{GHz}$ for the fractal structure with its four iterations IT0, IT1, IT2, and IT3. We can note that simulated values are lower than measured ones for antennas A0 and A1.

We conjecture that the irregular boundary increases the interaction between the localized modes, which may increase the gain and the efficiency of the antennas (see Table 1). The peak gain can reach $3.5 \mathrm{dBi}$ at $5.25 \mathrm{GHz}$ for IT0 and $4.8 \mathrm{dBi}$ at 5.28 GHz for IT1. The obtained gain and efficiency decrease when the complexity of the antenna nervures becomes much higher. In fact, if we increase the complexity of the antenna geometry by considering more details from the original image, this will give us more radiating elements of different lengths which are irregularly distributed. These elements act as a set of random dipoles radiating in all directions, which leads to cancellation of many elementary-radiated electric fields, and affect the efficiency of the structure and then its gain.

Finally, we can conclude that the main common point between all fractal antennas (deterministic and random) is their multiband behavior; however, irregular fractal structures inspired from nature such as the proposed leaf antenna or the fluid-jet antenna [11] offer more resonating peaks than deterministic fractal antennas such as the Sierpinski triangle antenna [4] and Koch monopole antenna [5], which is very useful for RF coding applications.

\section{Conclusion}

In this work, a variety of 2D fractal tree-leaf antennas was designed following a specific iteration procedure. The effects of the contours and the nervure networks of these 


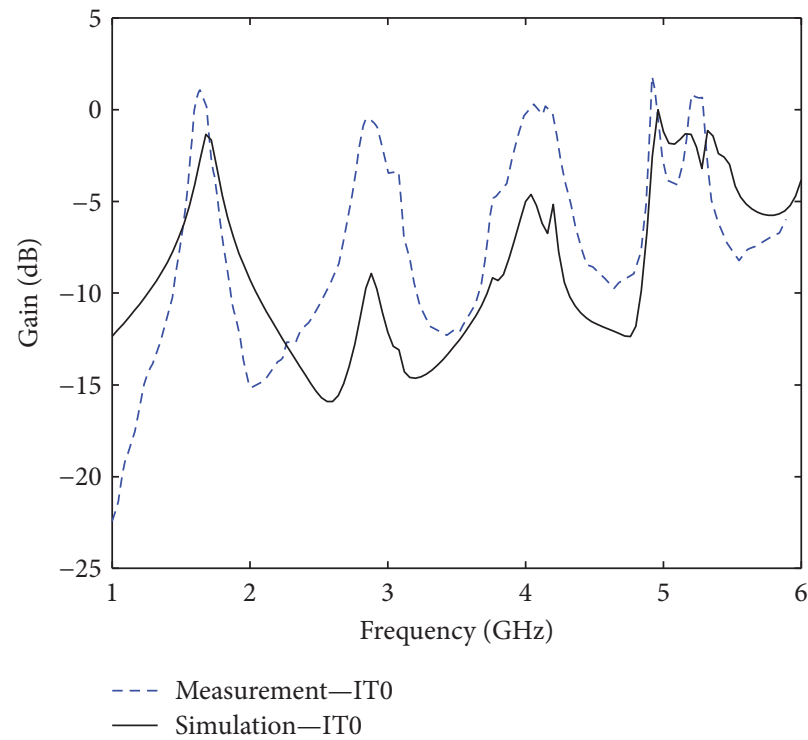

(a)

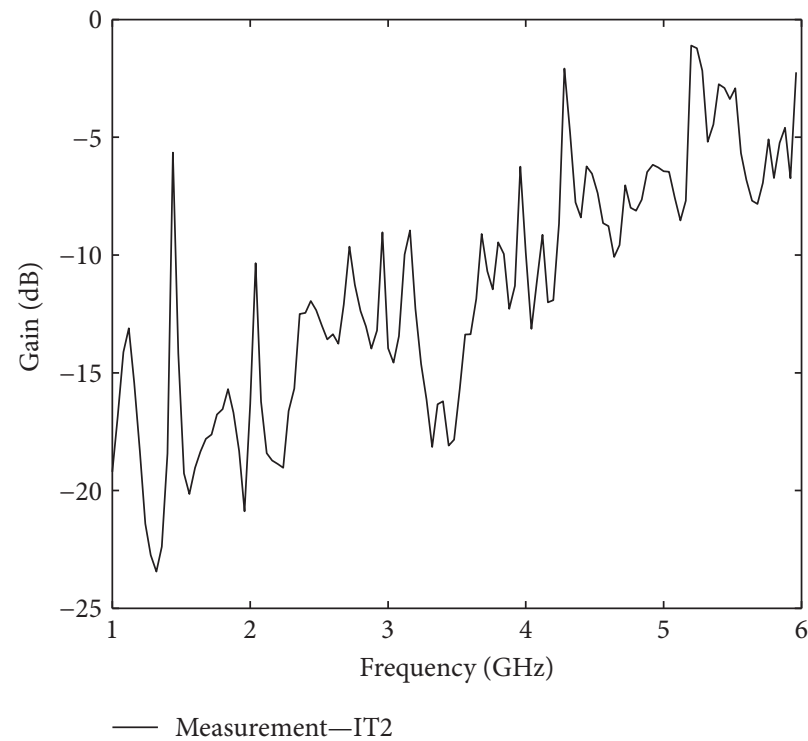

(c)

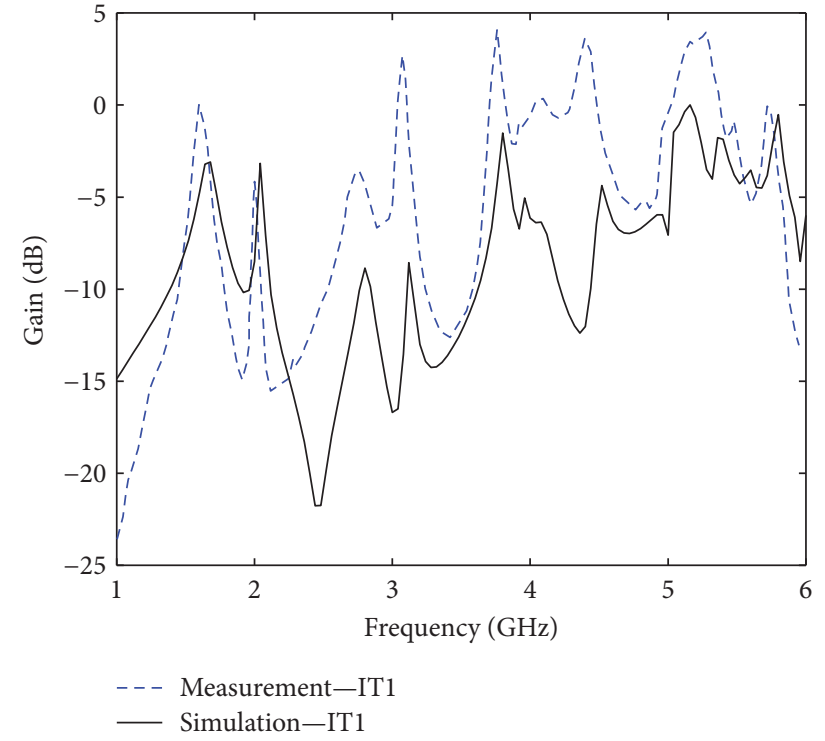

(b)

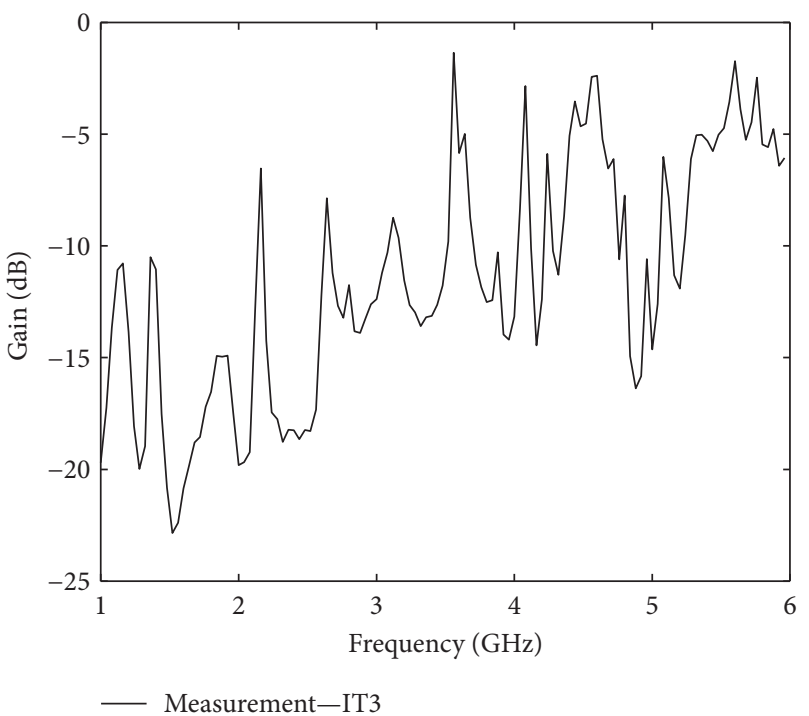

(d)

FIGURE 7: Simulated (black) and measured (blue) gain values for antennas: (a) A0, (b) A1, (c) A2, and (d) A3.

fractal antennas on their radiation characteristics were investigated and analyzed. The number of obtained narrow bands depends on the complexity of the irregular contour and the nervure network. A good input impedance matching and stable radiation properties were achieved. We conclude with the observation that this type of antenna structure would be suitable for the design of RF tags with unique electromagnetic signature especially if the return loss response is complex and composed of a great number of randomly distributed resonances.

\section{Conflicts of Interest}

The authors declare that there is no conflict of interests regarding the publication of this article.

\section{Acknowledgments}

This work was funded by the Deanship of Scientific Research (DSR), King Abdulaziz University, Jeddah, Saudi Arabia under Grant no. 135-420-D1435. The authors, therefore, acknowledge with thanks the technical and financial support provided by DSR. The authors are also grateful for the help of Dr. B. Blonder from the Department of Ecology and Evolutionary Biology, University of Arizona, USA for his helpful data dealing with the image acquisition and processing of the leaf image.

\section{References}

[1] B. Mandelbrot, The Fractal Geometry of Nature, W. H. Freeman, New York, NY, USA, 1983. 
[2] D. H. Werner, R. L. Haupt, and P. L. Werner, "Fractal antenna engineering: the theory and design of fractal antenna arrays," IEEE Antennas and Propagation Magazine, vol. 41, pp. 37$59,1999$.

[3] J. P. Gianvittorio and Y. Rahmat-Samii, "An overview of fractal antenna engineering research," IEEE Antennas and Propagation Magazine, vol. 44, pp. 20-36, 2002.

[4] J. Romeu, R. Pous, and A. Cardama, "On the behavior of the Sierpinski multiband fractal antenna," IEEE Transactions on Antennas and Propagation, vol. 46, pp. 517-524, 1998.

[5] C. Puente, J. Romeu, and A. Cardama, "The Koch monopole: a small fractal antenna," IEEE Transactions on Antennas and Propagation, vol. 48, pp. 1773-1781, 2000.

[6] C. Puente, J. Claret, F. Sagues, J. Romeu, M. Q. Lopez-Salvans, and R. Pous, "Multiband properties of a fractal tree antenna generated by electrochemical deposition," Electronics Letters, vol. 32, pp. 2298-2299, 1996.

[7] H. Rmili, J. L. Miane, J. M. Floch, and H. Zangar, "Design of multi and wideband 3D fractal monopole antenna for wireless operations in L- and S bands," Microwave and Optical Technology Letters, vol. 51, no. 8, pp. 1976-1979, 2009.

[8] H. Rmili, O. Mrabet, J. M. Floch, and J. L. Miane, "Study of an electrochemically-deposed 3D-fractal tree-monopole antenna," IEEE Transactions on Antennas and Propagation, vol. 55, pp. 1045-1050, 2007.

[9] H. Rmili, J. M. Floch, and H. Zangar, "Experimental study of a 2D-irregular fractal-jet printed antenna," IEEE Antennas and Wireless Propagation Letters, vol. 8, pp. 328-331, 2009.

[10] B. Blonder, C. Violle, and B. J. Enquist, "Assessing the causes and scales of the leaf economics spectrum using venation networks in Populus tremuloides," Journal of Ecology, vol. 101, pp. 981-989, 2013.

[11] H. Rmili, D. Oueslati, L. Ladhar, and M. Sheikh, "Design of chipless RFID tags based on natural fractal geometries for security applications," Microwave and Optical Technology Letters, vol. 58, no. 1, pp. 75-82, 2016.

[12] D. Oueslati, H. Rmili, C. Dumouchel, and J. M. Floch, "Numerical analysis of radiation properties of 2D-irregular fractal-jet printed antenna," in The 8th European Conference on Antennas and Propagation (EuCAP 2014), pp. 2165-2169, The Hague, Netherlands, April 2014. 


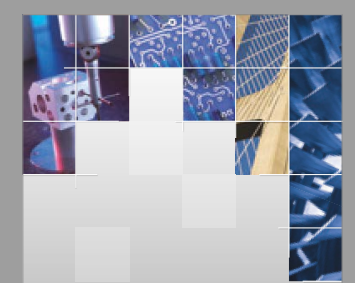

\section{Enfincering}
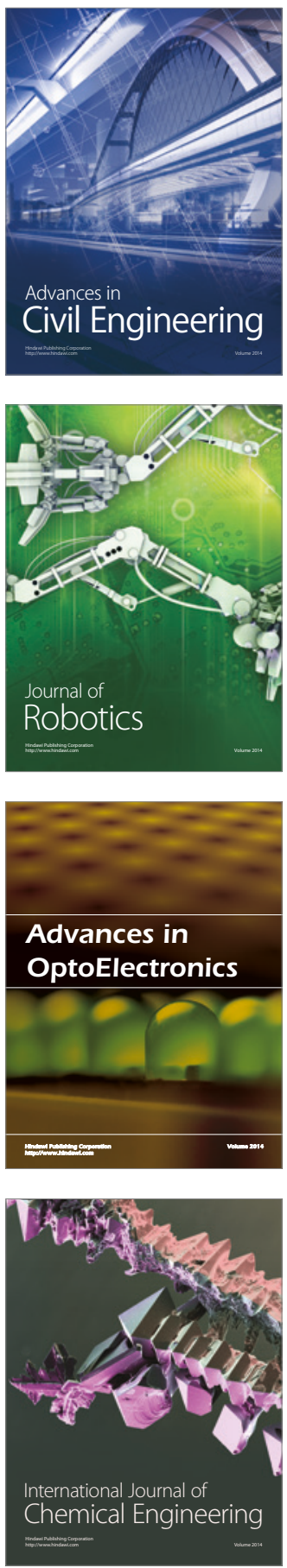

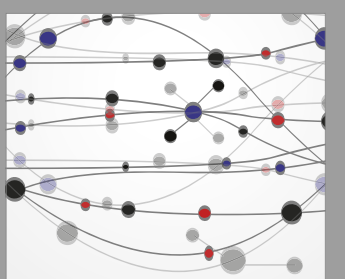

The Scientific World Journal

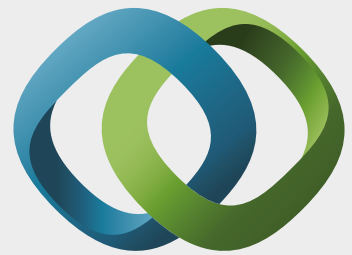

\section{Hindawi}

Submit your manuscripts at

https://www.hindawi.com
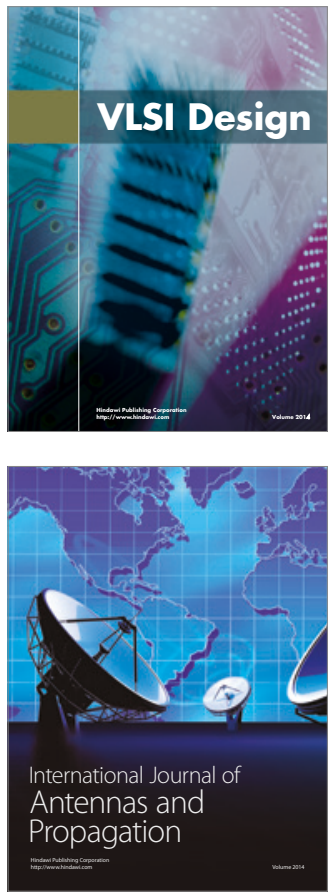

\section{Rotating}

Machinery
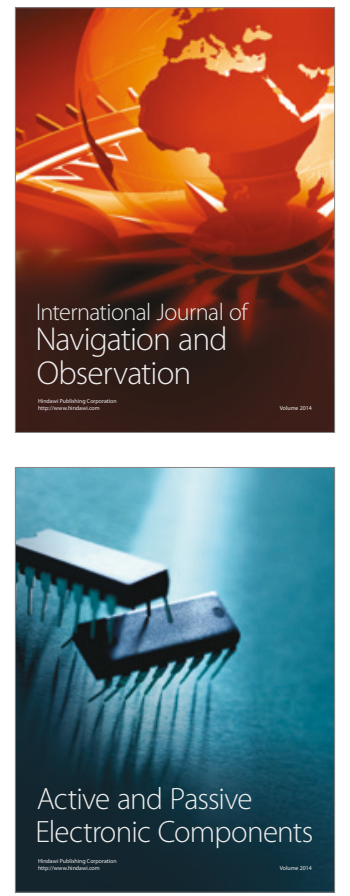
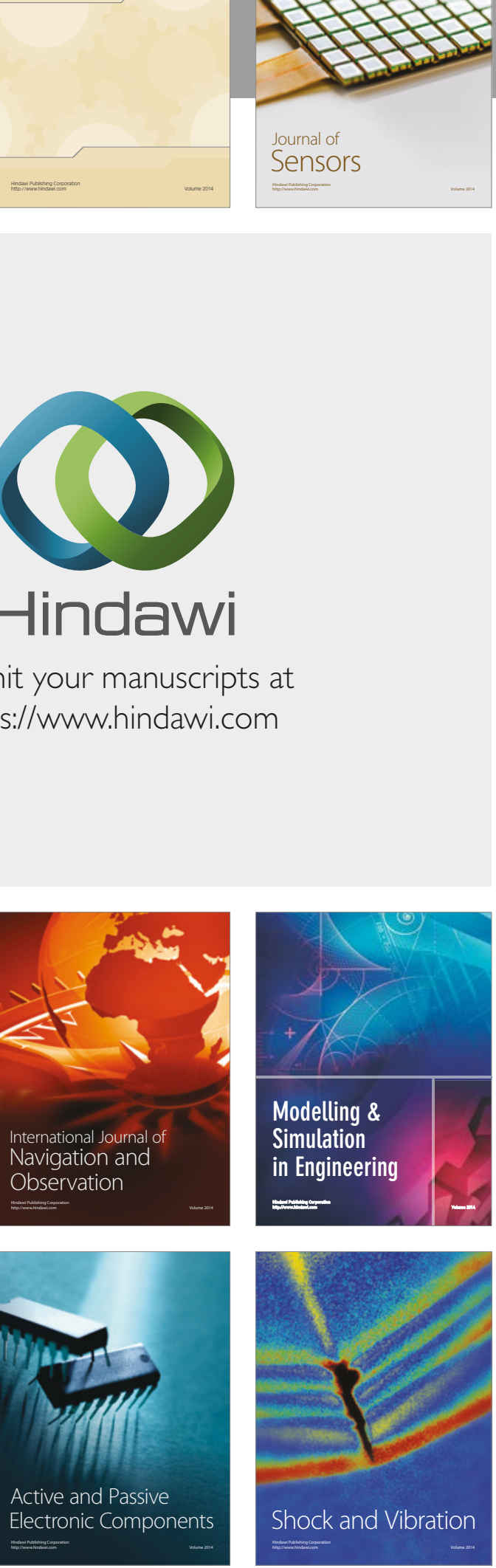
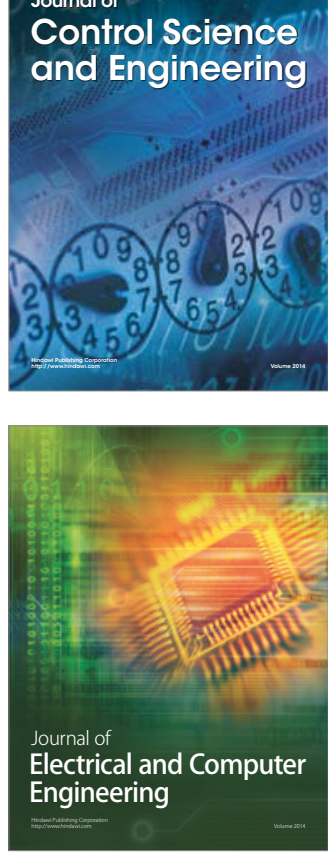

Distributed

Journal of

Control Science

and Engineering
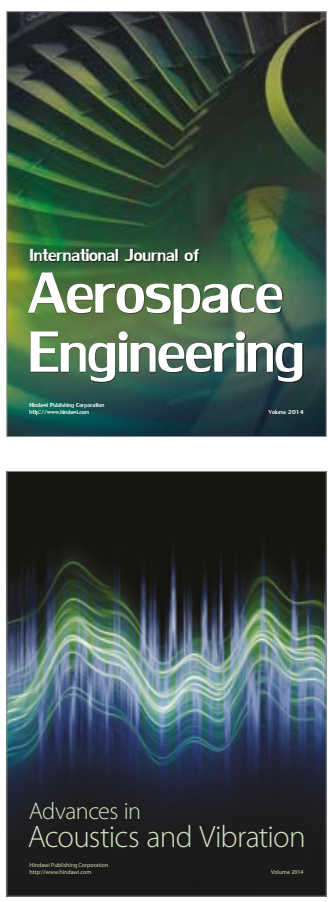

Sensor Networks 